Revue d'histoire de l'Amérique française

REVUE D.HISTOIRE DE L'AMÉRIQUE FRANÇAISE

\title{
Papineau et la rivalité Québec-Montréal (1820-1840)
}

\section{Fernand Ouellet}

Volume 13, numéro 3, décembre 1959

URI : https://id.erudit.org/iderudit/301984ar

DOI : https://doi.org/10.7202/301984ar

Aller au sommaire du numéro

Éditeur(s)

Institut d'histoire de l'Amérique française

ISSN

0035-2357 (imprimé)

1492-1383 (numérique)

Découvrir la revue

Citer cet article

Ouellet, F. (1959). Papineau et la rivalité Québec-Montréal (1820-1840). Revue

d'histoire de l'Amérique française, 13(3), 311-327.

https://doi.org/10.7202/301984ar d'utilisation que vous pouvez consulter en ligne.

https://apropos.erudit.org/fr/usagers/politique-dutilisation/ 


\section{PAPINEAU ET LA RIVALITE QUÉBEC-MONTRÉAL (1820-1840)}

Essentielle à la compréhension de l'histoire politique du Bas-Canada et à l'étude psychologique des Papineau, la correspondance de L.-J. Papineau fournit également des indices précieux de la mentalité des classes moyennes canadiennes-françaises au $19^{\circ}$ siècle. Cette correspondance permet aussi de mettre l'accent sur l'importance de la rivalité Québec-Montréal dans l'évolution des conflits qui opposèrent, de 1820 à 1840, les classes moyennes canadiennes-françaises aux commerçants et aux bureaucrates anglo-saxons. Profondément «montréaliste», Papineau a néanmoins déploré à plusieurs reprises les conséquences funestes de ces dissensions sur la stabilité du parti patriote. Il mettait alors l'accent sur le conservatisme et l'opportunisme des hommes politiques de Québec. Même si le témoignage de Papineau sur la société québécoise demande d'être nuancé, il demeure au plus haut point révélateur des tensions permanentes qui affectèrent le parti patriote. L'idéologie nationaliste avait un caractère beaucoup plus abstrait et idéaliste que celle qui inspirait le parti bureaucrate et, par une contradiction assez singulière, elle servait, pour une large part, à défendre des intérêts immédiats, locaux ou particuliers. Dans ces conditions, on peut comprendre le rôle capital des rivalités personnelles, des luttes de clans et des sentiments locaux sur le destin d'un groupement politique qui, tout en voulant assurer la permanence des cadres féodaux, se donnant pour mission d'édifier dans le Bas-Canada un État national canadien-français doté d'une structure laïque et démocratique. Au contraire, le parti bureaucrate, qui visait à l'unification économique de la vallée du SaintLaurent, n'avait pas à surmonter les mêmes problèmes. Certaines tensions existaient parmi les bureaucrates; mais les marchands, servis par une idéologie axée sur un programme écono- 
mique à long terme, manifestèrent une profonde solidarité en face du parti patriote. ${ }^{1}$

C'est dans cette perspective d'action nationale et démocratique que le témoignage de Papineau sur la société de Québec prend sa plus grande signification. Dans l'analyse de ce témoignage, il faut aussi tenir compte de la conscience «montréaliste » de Papineau et même de certains traits de sa personnalité. Enfin il importe de préciser rapidement les fondements de la rivalité Québec-Montréal.

\section{Les fondements de la rivalité Québec-Montréal}

La rivalité Québec-Montréal n'était pas un phénomène nouveau au début du $19^{\mathrm{e}}$ siècle. Elle datait du $17^{\mathrm{e}}$ siècle. Ce sentiment d'opposition entre les deux noyaux urbains de la NouvelleFrance tenait sans doute à des différences réelles. Québec n'avait-il pas été, depuis le $17^{\mathrm{e}}$ siècle, le port de mer, la capitale administrative et religieuse, la résidence de l'élite militaire et le centre de la vie sociale? Dominant une région agricole, Montréal avait dû sa fortune au commerce des fourrures lequel avait marqué davantage sa stratification sociale. Montréal était très tôt devenu la porte de l'Ouest. Les ecclésiastiques des deux villes n'étaient pas non plus demeurés indifférents au développement de l'esprit local. Ainsi la fierté respective de ces deux groupements urbains constituait un facteur non négligeable dès qu'il s'agissait d'appliquer un programme d'action commune. Telle était la conséquence inévitable de l'introduction du régime parlementaire et de l'apparition des aspirations nationales. Au lieu de diminuer le chauvinisme local, les transformations économiques et démographiques qui affectèrent Québec et Montréal,

1 « Mais qu'il est difficile de conserver du concert et de l'union entre un grand nombre d'hommes, qui ne sont pas organisés, comme les agens du gouvernement, à agir d'après l'impulsion d'un petit nombre d'hommes, vers une direction unique. Il $\mathrm{y}$ a toujours quelque semence de division entre nous prête à éclater. La rivalité entre Québec et Montréal est toujours prête à renaître, par la faiblesse de quelques-uns des Messieurs d'ici. L'étourderie de Bédard et l'artifice de Mr Vanfelson nous tiennent toujours aux bords de l'abîme. 》 Lettre à sa femme (16 nov. 1835). RAPQ (1953-55), $364 \mathrm{~s}$. 
au cours de la première moitié du $19^{e}$ siècle, contribuèrent plutôt à le renforcer.

Pendant la première moitié du $19^{\mathrm{e}}$ siècle, le visage de Québec se modifia radicalement. ${ }^{2}$ Le commerce des fourrures cessa alors de définir le rythme de l'économie canadienne. Il fut remplacé par l'exploitation forestière, les céréales, la potasse et la construction navale. L'augmentation de la population et du pouvoir d'achat se traduisirent par un accroissement énorme des importations. Québec s'affirma alors comme le port de mer du Canada. Sous le régime français, le nombre des arrivages avait varié de 4 à 20 navires par année; de 1760 à 1785, il s'établissait de 20 à 60 ; en 1795, 128 navires avaient accosté au port de Québec et, en 1802, 211. De 1808 à 1819, les arrivages avaient été portés de 334 à 630 vaisseaux. En 1828, ils atteignaient 701 et, enfin, de 1830 à 1840, la moyenne annuelle était de 1,000 navires. Il s'agissait d'une véritable révolution qui s'inscrivait aussi sur les plans démographique et social.

La population de Québec passa de 15,237 habitants, en 1819, à 26,000 en 1830 . Le caractère ethnique de cette population en fut nettement transformé. En 1819, plus de 4,200 Anglo-saxons. Onze ans plus tard, les seuls effectifs irlandais se montaient à 7,000 personnes. En 1851, 35.5\% de la population de Québec était anglo-saxonne. Ces chiffres ne comprenaient pas une énorme population flottante. En plus des immigrants qui arrivaient au port de Québec, de 8,000 à 15,000 marins s'y arrêtaient pendant la saison de navigation. La construction navale, qui donnait de l'emploi à un grand nombre de journaliers et de paysans canadiens-français, pendant l'hiver, entraînait un afflux saisonnier important. En 1840, une grève de charpentiers de navires impliquait 6,000 ouvriers. Les recensements ne tiennent pas compte, croyons-nous, de la garnison militaire qui englobait, selon les années, de 500 à 3,000 hommes. Enfin le commerce du bois amenait à Québec, au cours de l'été, un nombre toujours plus grand d'employés forestiers, chargés de diriger les radeaux, et

2 Raoul Blanchard, L'est du Canada français - «Province de Québec 》 (5 vol., Québec, 1935-1948) : «Québec — Esquisse de géographie urbaine », II : 157-289. 
de commerçants de bois. Cette population flottante donnait à Québec un caractère propre aux grandes villes portuaires. L'arrivée des marins y engendrait une véritable frénésie.

Résidence du Gouverneur-Général de plusieurs provinces nord-américaines de l'Angleterre, Québec possédait un fort contingent de fonctionnaires à majorité anglo-saxons. Enfin la capitale provinciale était le centre religieux de l'Amérique du Nord britannique.

En raison de la concentration du grand commerce et de l'industrie aux mains des Anglo-saxons et de la situation prédominante de ces derniers dans l'administration, la ville de Québec avait l'allure d'une ville britannique. Sur le plan social, l'élément anglais donnait le ton. Les ouvriers, les artisans, les petits commerçants et les membres des professions libérales d'origine française dépendaient plus ou moins des négociants anglais et du gouvernement sur le plan du travail. L'augmentation excessive de la classe des petits marchands et de celle des notaires, des avocats et des médecins accentuait cette dépendance.

La situation économique des professions libérales et des ouvriers canadiens-français de la ville de Québec rend compte à la fois d'une dépendance et d'un mouvement d'hostilité à l'égard de la population anglaise et, en particulier, des Écossais. Les crises, en réduisant l'activité économique, atteignaient plus durement l'élément canadien-français et les Irlandais. Les différences raciales jouèrent un rôle essentiel dans les relations entre le capital et le travail. C'est pourquoi les phases de tensions politiques, sociales et raciales reproduisent les fluctuations de l'économie. Si, en période de difficultés économiques, le parti patriote pouvait assez facilement obtenir le support des ouvriers et des petits marchands, en revanche, la reprise des affaires rendait souvent impossible une action concertée. Quoique plus complexe, la réaction des professions libérales suivait dans une large mesure le jeu de la conjoncture économique et politique. C'est ce qui faisait dire à Papineau: "Québec s'augmente et s'enrichit incomparablement plus que Montréal, mais dans le commerce, 
non dans la Société canadienne $\gg^{3}$ Il pouvait difficilement comprendre pourquoi le patriotisme des québécois comportait tant de facteurs variables.

Pourtant Montréal avait subi la même évolution que Québec. ${ }^{4}$ Sa population était passée de 16,000 habitants, en 1820 , à 26,000 en 1825. En 1844, elle se chiffrait à 44,485 personnes. Fait étonnant, si on s'en tient aux remarques contraires de Papineau, la population d'origine britannique y était plus considérable qu'à Québec. Dès 1820, 37\% de la population était anglo-saxonne; en $1825,43 \%$; en 1831, $57 \%$ et, vingt ans plus tard, $54 \%$. Comme dans la capitale, les britanniques détenaient le contrôle du commerce, de l'industrie et des institutions de crédit. Mais l'économie de Montréal était mieux articulée à l'arrière-pays que Québec. Elle commandait une région agricole plus productive et plus peuplée. Les classes moyennes canadiennes-françaises y étaient plus nombreuses, plus près des populations rurales et elles manifestaient une plus grande solidarité. Les problèmes agraires étant plus aigus et la présence anglo-saxonne plus intense, les classes paysannes y étaient plus sensibles aux objectifs nationalistes. De même les contacts avec les États-Unis s'y faisaient plus nombreux que dans la région de Québec. En conséquence les préventions contre les idées démocratiques s'en trouvaient diminuées. Lorsque Papineau parle de Montréal, il réfère constamment à ce groupement urbain lié à un arrière-pays immédiat adonné à la vie agricole et imbu de solides traditions familiales. Le Montréal des gros négociants lui échappait.

Montréal était aussi le point de transit qui donnait accès par le Saint-Laurent au Haut-Canada alors en plein développement. C'était la ville pionnière et celle de l'avenir. C'est pourquoi les négociants de Montréal les plus actifs étaient davantage intéressés par le ravitaillement des nouvelles régions de colonisation des Grands Lacs et par les céréales qui en provenaient. Leurs capitaux étaient aussi investis dans l'exploitation forestière. Ils étaient, à cette époque, les principaux promoteurs de la canali-

3 Papineau à sa femme, 28 janvier 1830, RAPQ (1953-55), 275.

4 Raoul Blanchard, L'ouest du Canada français - (5 vol., Montréal) « Montréal: esquisse de géographie urbaine », IV: 171-382. 
sation du Saint-Laurent et du développement portuaire de Montréal qui débuta en 1832. Désormais le port de Montréal allait devenir le centre canadien d'exportation des céréales.

Ainsi Montréal faisait figure de ville plus continentale. Le rythme de la vie y était plus lent. En dépit de son infériorité numérique, la population canadienne-française y formait un bloc compact solidaire de la zone rurale, ce qui était beaucoup moins vrai pour Québec. Malgré une population flottante d'immigrants qui se rendaient dans le Haut-Canada et de bûcherons, Montréal avait une physionomie plus calme. Si les classes ouvrières se trouvaient dans une situation semblable à celles de Québec visà-vis des employeurs anglo-saxons, en revanche, les petits marchands et les professions libérales y étaient plus indépendants des capitalistes et du gouvernement. Ces facteurs rendent compte de l'influence plus prononcée de l'idéologie nationaliste et démocratique dans la région de Montréal.

\section{Papineau et la société québécoise}

Papineau n'a jamais aimé la ville de Québec. Enraciné à la région de Montréal par ses attaches familiales, par les traditions rurales et par ses conceptions de la vie sociale, il avait édifié sa carrière politique en s'appuyant davantage sur la moyenne bourgeoisie de Montréal. D'ailleurs sa pensée politique et sociale trouvait une résonance plus profonde dans l'ouest du Bas-Canada. Les cadres du parti patriote reflétèrent même ses affections familiales et locales. Il y avait là des raisons d'aiguiser la susceptibilité des Québécois qui avaient dominé le parti canadien-français à l'époque du premier Canadien. Peu après son élection comme orateur de la Chambre d'Assemblée, Papineau n'était-il pas, en certains milieux, qualifié de québécois ? En raison de l'importance qu'il accordait à la vie de famille, il aurait été normal que Papineau songeât à se fixer à Québec. En 1826, il écrivait à sa femme:

Qu'attends-tu à me donner des détails de Montréal ? je fais réflexion parfois que, quoique durant la Session, il me serait bien agréable d'être ici en famille, il me serait bien désagréable d'y vivre hors 
le tems des Sessions. Hors Mr Neilson, avec qui je me trouve des principes communs et des études analogues, je vois en général si peu d'indépendance de caractère, si peu d'esprit ou, quand il $\mathrm{y}$ en a, des esprits si légers; une disposition si générale à flatter lâchement les grands abus, comme moyen d'avancement, que je n'y connais aucune société dans laquelle je m'y plairais. Nous avons à Montréal où elles sont pourtant trop rares, plus de personnes honnêtes et instruites qu'il y en a ici. Mon voisin [Jacques] Viger qui a le goût des beaux arts, qui t'aura régalé dimanche dernier de la belle gravure de $\mathrm{Mr}$ de Salaberry; qui chante et cause si bien, que la société dans laquelle il vit rend patriotique, vivrait ici dans un autre cercle où il se perdrait. ${ }^{5}$

Non, Papineau le rigide républicain, ami des livres et de la vie campagnarde, ne pouvait accepter de résider dans un «lieu de frivolité et de dissipation comme Québec ${ }^{6}$ Certes il avait souvent rencontré des hommes estimables et capables de conversation solide mais leur patriotisme laissait à désirer. L'Évêque de Québec, dont « la conversation [était pourtant] une source intarissable d'informations et d'observations agréables et utiles », manquait cependant devant le gouvernement de fermeté "jusqu'à la fin du chapitre ». ${ }^{7}$ Papineau se plaisait aussi à rencontrer les prêtres du Séminaire; mais il arrivait souvent que « des hommes graves et éclairés, par le manque d'usage du monde et l'habitude de ne vivre qu'avec des enfants, y dépar[aient] la conversation par des enfantillages $\gg .8$ Il avait aussi très bien connu Duchesnay; mais ce dernier faisait partie de l'ancienne

5 Papineau à sa femme (2 mars 1826), RAPQ (1953-55), 238. En 1832, il revenait sur la question: «Parfois je regrette bien de n'être pas venu il y a quelques années m'établir à Québec. D'un autre côté qui sait si la mauvaise compagnie ne m'y aurait pas gâté ? Si je n'y serais pas devenu un souple courtisan? A l'heure qu'il est il n'y a plus semblable risque. Il y a assez longtems que je marche la tête haute, que je ne craindrais pas de m'accoutumer à monter les escaliers du Château St Louis comme la Scala Santa à Rome, où les dévôts et dévotes ont de leur front et genoux usé la pierre. Je marcherais ici libre comme là-bas et je ne me séparerais pas si longtems de ma chère Maman ... Ibid., 325.

$6 \mathrm{P}$. ̀̀ sa femme (20 déc. 1820$), \mathrm{RAPQ}(1953-55), 192$.

$7 \mathrm{P}$. à sa femme ( 7 janv. 1821), Ibid., 195.

$8 \mathrm{P}$. à sa femme (3 fév. 1825), Ibid., 218. 
noblesse domestiquée par les Gouverneurs. ${ }^{9}$ La moyenne bourgeoisie lui apportait aussi peu de satisfaction. Louis Plamondon, par exemple, son condisciple au Séminaire de Québec et ami, «a autant et plus d'esprit que presqu'aucun autre homme que je connaisse, mais nous sommes aux antipodes l'un de l'autre en politique, et cela fait que nous ne pourrions pas avoir des liaisons aussi intimes qu'avec mes amis de Montréal. Quant à la plupart des hommes publics, plus on suit leurs vues et leurs actes plus on y trouve à redire; un honnête homme est journellement forcé de les accuser en Chambre et l'on ne doit ni ne peut les voir individuellement ensuite. ${ }^{10}$ Papineau ne pouvait non plus se plaire en compagnie des négociants anglais dont il dénonçait avec assurance « l'avarice, la cupidité et l'esprit de domination ». Selon lui, ils étaient incapables de concilier une recherche légitime du gain avec les exigences de la vie familiale et avec une saine conception bourgeoise de la vie. Papineau voyait réellement la ville de Québec comme une enclave britannique dans un Bas-Canada canadien-français par ses institutions, sa langue et ses coutumes. Centre de la corruption administrative et de l'égoïsme mercantile, Québec était aussi selon lui, peu favorable au développement d'un patriotisme éclairé et désintéressé. La capitale provinciale lui apparaissait même comme foyer de désintégration pour la culture nationale des Canadiens français. En 1828, il écrivait à sa femme:

Ces jours derniers, la Chambre s'est plusieurs fois ajournée à bonne heure, et cela a fait que j'ai répondu à plusieurs invitations à diner, toutes Anglaises. Eux seuls, dans Québec ont le ton et la fortune nécessaire pour recevoir. Il n'y a pas une seule maison canadienne qui le puisse faire. Les ressources du pays sont dévorées par les nouveaux venus, et quoique j'aie le plaisir de rencontrer parmi eux des hommes instruits, estimables, qui me voient aussi avec plaisir, la pensée que mes compatriotes sont

9 «Il a vécu sur sept à huit cens Louis par an, sans rien économiser et moitié de ce revenu provenait de places qui finissent avec lui. Voilà encore une des anciennes familles qui va tomber dans l'obscurité, puisqu'elle ne demeure qu'avec peu de fortune et nulle influence et éducation. $\gg$ P. à sa femme (17 févr. 1825), Ibid., 223.

$10 \mathrm{P}$. à sa femme (2 mars 1826), Ibid., 238. 
injustement exclus de participer aux mêmes avantages m'attriste au milieu de leur réunion, et me rendrait le séjour de Québec désagréable. Le mal n'existe pas au même degré à Montréal. ${ }^{11}$

Même si le témoignage de Papineau sur la société québécoise provenait, pour une part, d'un nationalisme intransigeant et d'un certain chauvinisme local, il reste que la rivalité QuébecMontréal a pesé lourdement sur la stabilité du parti canadienfrançais. La conscience québécoise s'est souvent insurgée contre la prédominance du groupe montréalais et contre Papineau, qui en était le chef.

L'élection de Papineau, en 1815, comme orateur de la Chambre, avait réduit l'influence des députés de Québec dans le parti. Jusqu'alors Panet avait limité le rôle de l'Orateur à la direction de la procédure parlementaire. Mais lors de son élection, Papineau était déjà reconnu comme le successeur de Bédard à la tête du parti canadien-français. Il continua, après 1815 , à assumer les deux fonctions. Mais Taschereau, Blanchet, Borgia et Bourdages pouvaient difficilement lui pardonner son élection obtenue grâce aux députés de Montréal et à un certain nombre de Québécois. Une coalition québécoise se forma pour déloger Papineau. En 1818, Papineau écrivait à son père:

Mais ce qui me choque ce sont les tracasseries que me suscite dans un pareil moment Mr Taschereau qui choule Mrs Borgia et Vanfelson à japper à tort et à travers. Ce Mr. plus ancien que moi au parlement est bien persuadé que je lui ai ravi le prix qui n'appartenait à son avis qu'au Gendre de Mr Panet. $^{12}$

Puis, en 1820, Bourdages, Blanchet et Cuvillier se firent les promoteurs d'une démocratisation plus poussée de la Chambre en favorisant la candidature de paysans. L'année suivante, ils proposèrent une loi pour la rémunération des députés. Papineau était alors opposé à cette mesure qui visait à lui enlever l'appui d'une partie de la députation. Le 15 février 1821, il écrivait à son père: 
Blanchet va enfin proposer d'accorder une compensation aux membres à laquelle il était autrefois opposé, et il le fait en flattant d'une manière ridicule la Campagne en leur disant que s'il y a eu tant de dissolutions du parlement c'est que la chambre n'était pas pleine de représentans de la Campagne, qu'il est honteux de voir que le quart de la représentation est composée d'avocats et de Notaires qui vivent du travail de l'habitant et que c'est celui-ci qui fait vivre tous les autres états qui doit maitriser la direction des affaires. ${ }^{13}$

En 1823, lorsqu'il fut question de déléguer Papineau en Angleterre, afin de faire échouer le projet d'union des Canadas, l'abbé Demers se montra d'abord réticent et il manifesta son inquiétude au sujet de l'avenir du parti canadien-français. Il craignait que les rivalités personnelles ne provoquent la dissolution du parti. Après le départ de Papineau, Vallières de SaintRéal le remplaça comme orateur. Sa mission terminée, Papineau, en dépit de prétentions contraires, tenait à reprendre ses fonctions. Mais Vallières n'était pas disposé à céder. Les années qui suivirent l'échec de l'Union, même si elles virent le triomphe définitif de Papineau comme chef du parti canadien-français, furent marquées par un renouveau des rivalités personnelles et de clans. Cette fois Bourdages et ses alliés s'attaquèrent à la «Sacrée clique » des Papineau et Viger qui dominaient les milieux politiques de Montréal et prétendaient mener les Québécois. ${ }^{14}$ Dégoûté, Papineau proclama que «l'avidité de Québec 》n'avait pas de bornes. Il écrivit à sa femme:

Les Canadiens se plaignaient avec raison de l'administration quand elle attendait la fin des sessions pour emporter en l'absence de la Majorité des membres quelques mesures favorites. Ils donnent aujourd'hui le même Scandale public. La cotterie de Québec, guidée par ses affections et liaisons de Société propose des actes de profusion du revenu, en dons pour Bouchette, Leblond, la Veuve Willing et autres; le font en foulant les règles aux pieds, c'est là le mal, et donnent le prétexte à mille autres folles

$13 \mathrm{P}$. à son père (15 févr. 1821), Ibid., 129.

$14 \mathrm{P}$. à sa femme (8 janvier 1825), RAPQ (1953-55), 212s. 
demandes et crient un peu contre les Montréalistes. Voilà le mauvais côté de la Question. ${ }^{15}$

En 1826, Papineau perdit deux appuis importants dans les personnes de Moquin et de Planté qui exerçaient une influence considérable sur les députés de Québec. Par contre le parti canadien-français fut réorganisé et devint le parti patriote supporté par La Minerve. D'ailleurs, à partir de 1826, Papineau pouvait compter sur l'appui presque total des populations paysannes. C'est la période de transition vers une nouvelle forme de nationalisme plus traditionaliste et lié à l'idéologie démocratique. Certes, les difficultés économiques et leurs conséquences sur la stabilité de certaines classes sociales étaient favorables à une orientation vers le radicalisme. Par contre l'instabilité économique affermissait plus encore le parti bureaucrate. Même si l'autorité de Papineau s'affirma davantage sur le parti patriote, en raison de son influence sur le peuple, les rivalités personnelles, les luttes de clans, la rivalité Québec-Montréal, celle entre les villes et les campagnes ne furent pas éliminées. Encore, en 1829, Vallières de Saint-Réal essayait de reprendre le prestige perdu. Mais l'événement le plus important de la période pour le parti patriote, fut la rupture de Papineau avec Neilson.

John Neilson avait appuyé le parti canadien-français depuis 1818. Son influence dans la région de Québec avait servi à renforcer le groupe nationaliste et réformiste. Possédant un meilleur équilibre émotif que son chef, Neilson, tout en demeurant au second plan, avait puissamment contribué à l'ascension de Papineau. En 1828, ce dernier lui écrivait:

Vous valez bien mieux que moi, vous qui avez de la sensibilité comme j'en ai, et qui avez de la force comme je n'en ai pas. Il n'est aussi personne que j'aime autant à voir comme vous; vous avez plus d'une fois raffermi mon courage chancelant. La perte de mes amis me livre à la mélancolie au point qu'elle me jetterait presque dans une entière et stupide inaction, qui est ma malheureuse inclination dominante. L'injustice faite à mon pays me tue, m'agite au point de n'être pas toujours en état de ne

15 P. à sa femme (12 mars 1825), Ibid., 229. 
prendre conseil que d'un patriotisme éclairé, mais plutôt de la colère et de la haine contre ses oppresseurs. $^{16}$

La rupture de Papineau et de Neilson provenait, selon toute apparence, de motifs idéologiques. Neilson était un libéral et, comme tel, il était disposé à supporter un programme de réformes politiques et à défendre l'égalité des groupes raciaux. Mais il se refusait à appuyer une pensée démocratique placée au service d'intérêts purement nationalistes. En 1833, Neilson supporté par quelques députés devenait l'adversaire résolu de son ancien chef. Cet événement se compliqua d'une recrudescence de l'esprit local chez les députés de Québec. Au mois de janvier 1834, Papineau écrivait à sa femme:

Il y aura donc Session; - il est impossible qu'elle ne soit pas accompagnée de mille et mille actes de faiblesses, puisque le principe de cette détermination part chez Neilson et ses amis, d'une détermination évidente de soutenir et conserver la présente administration et, de la part des patriotes québécois, de la détermination apparente de se faire auprès d'elle préférer à Neilson. Celui-ci est bien supérieur aux autres; mais leurs divisions sauveront le pays; la fixité et l'opiniâtreté avec lesquelles le premier tient à son système déplaira bien vite au pays; il se trouvera seul avec les Anglais des townships et dès lors ne saurait avoir longtems de l'influence - Les autres sont trop légers, tour à tour complaisans pour le gouverneur, ou emportés contre lui, pour de légers torts s'ils en souffrent personnellement. Leur cœur est pour le pays - Mais un peu de vanité, l'âme de tous les mouvemens que l'on se donne dans Québec pour paraître au Château d'un doigt plus grand que sa voisine; et des intrigues qu'ils ne savent pas démêler leur ont fait faire des actes de complaisances dans lesquels ils ne savent pas et ne veulent pas toujours persister ... ${ }^{17}$

16 P. à Neilson (9 janv. 1828), APQ, P-B: 509a.

$17 \mathrm{P}$. à sa femme (12 janvier 1834), RAPQ (1953-55), 338s. Il ajoutait: «Au Bal du 31, Madame la Mairesse a eu tous les honneurs; toutes les Canadiennes ont eues tous les honneurs: les Aide-de-Camp leur ont bien dit que le Gouverneur chérissait par dessus tous, les Canadiennes. Comment une femme ainsi flattée, ainsi coiffée, ne finirait-elle pas par aussi coiffer le mari. 》 
$\mathrm{Au}$ début de l'année 1834, l'attitude des députés québécois mettait un obstacle insurmontable à la ligne de conduite tracée par Papineau. De même l'année 1834 fut marquée par une grande détresse commerciale, industrielle et agricole. Les classes ouvrières (Canadiens français et Irlandais) et les classes paysannes se trouvaient dans une situation particulièrement pénible. Le moment était venu pour Papineau d'agir. En refusant de siéger, les députés placeraient l'Exécutif devant un dilemme. Si le gouvernement acceptait le défi de l'Assemblée et suspendait les travaux publics, faute d'un vote de la législature, la situation économique en serait aggravée. Il devrait alors faire face à une population montée à qui on avait inculqué l'idée que les capitalistes anglais et l'administration étaient les responsables de tous les malaises économiques. $\mathrm{Si}$, au contraire, le gouvernement s'emparait des subsides afin de réduire les effets de la crise, il était évident que Papineau pouvait encore soulever l'opinion publique au nom des principes parlementaires. Mais les députés de Québec tenaient à avoir une Session. Le 21 janvier Papineau écrivait à sa femme:

Mais notre politique après des désertions et des apostasies aussi scandaleuses que celle que nous voyons depuis une couple d'années, quoique l'administration soit la plus incapable que nous ayons jamais eue mais parce qu'elle est en même temps la plus intrigante et la plus corrompue qu'il soit possible d'imaginer, et que c'est à deniers comptans qu'elle achète non pas les consciences de ces déserteurs, car personne ne peut donner ce qu'il n'a pas, mais leurs suffrages; devient si vacillante, si incertaine, que l'on ne peut que s'affliger sans savoir où trouver le remède. Le dessous des cartes, c'est que Parent l'Imprimeur du Canadien, maîtrise Bédard et Huot, et ceux-ci, une partie des membres de Québec. Qu'il lui est dû $£ 1200$ à $£ 1500$ pour impression, que s'il n’y a pas de Session, il court le risque de ne pouvoir soutenir un pareil échec, que peut être sa feuille tombe; qu'ils ne sont pas des agens libres et désintéressés; que par ces circonstances le pays est déservi par ses amis, bien plus dangereusement qu'il ne l'est par ses ennemis; qu'ils veulent une session à tout prix; que 
l'administration connaît cette turpitude et leur arrachera mille actes de faiblesse, puis trouvera encore moyen de se venger d'eux. Je leur ai donné mes raisons et mes motifs de n'avoir pas de Session avec assez de force et de détail et de prévision de tout ce qui est arrivé depuis, qu'ils craignent plus que quoique ce soit au monde que je ne dise les mêmes choses publiquement, et que dès les premiers jours ils sont convenus d'adopter la tactique de n'avoir que le moins qu'ils pourraient de comités généraux, que quelques uns ont eu le patelinage d'en convenir, mais de dire que c'était par le respect et l'amitié qu'ils me portaient pour ne pas me laisser en minorité, vu que tout le District de Québec était en famine et en détresse, ne pouvait se passer de Session, qu'ils alloient pourvoir à leurs pressans besoins, puis après songeraient, avec infiniment de force et de courage à défendre leur honneur, au quinze février prochain. ${ }^{18}$

Les 92 résolutions furent donc préparées et présentées par suite du refus des députés de Québec d'adhérer à cette ligne de conduite préconisée par Papineau. En 1835, Papineau tenta à nouveau d'entraîner les députés de Québec à refuser une session. Le 2 mars, il écrivait à sa femme :

A Québec l'on veut d'une Session à tout prix. Avant l'ouverture du Parlement, il y avait eu beaucoup d'intrigues, pour ne pas se laisser mener, disaient-ils, par les gens de Montréal. Berthelot, l'un de ceux qui avaient été le plus dans des propos de ce genre, est heureusement dominé par Lafontaine. Il est très fermement Uni aux prétendus Meneurs, ainsi que le Dr Blanchet et Besserer. Ce sont Bédard, Huot, Caron et Vanfelson qui sont aigris; qui ressuscitent parmi les habitants les distinctions de localités. ${ }^{19}$

La défiance et le «refroidissement entre les patriotes de Québec et ceux de Montréal ${ }^{20}$ se maintint pendant toute l'année 1835. Au cours du mois de mars, il fut question d'envoyer un

18 P. à sa femme (21 janv. 1834), Ibid., 343s.

$19 \mathrm{P}$. à sa femme (2 mars 1835), Ibid., 356. Voir aussi la lettre du 26 février. Ibid., 354.

$20 \mathrm{P}$. à sa femme (14 mars 1835), Ibid., 359. 
agent en Angleterre pour défendre le point de vue de la Chambre d'Assemblée. Les patriotes de Québec virent dans cette mesure une occasion favorable pour détrôner Papineau. Le 5 avril, ils lui proposèrent de se rendre en Angleterre. Ce dernier ne fut pas dupe des motifs réels qui inspiraient leur démarche. Aussi la réponse de Papineau traduisait-elle une profonde inquiétude et un certain malaise. En déclinant l'honneur et la confiance qu'on lui faisait, il éprouva alors le besoin de refaire l'histoire du Bas-Canada depuis 1820 afin de démontrer l'inopportunité de cette délégation. ${ }^{21}$ Une lettre de Boucher-Belleville, montréalais, analysait les conséquences probables du départ de Papineau.

Nous différons entièrement de sentiment à l'égard de l'envoi de Mr Papineau en Angleterre. Je crois que ni le grand homme ni aucun autre ne peuvent faire de bien en Angleterre, où il est visible qu'on ne veut pas nous rendre justice. D'ailleurs qui ne voit que Lord Aylmer immédiatement après le départ de P.[apineau] ne se hâterait de rassembler la Chambre. Eh ! qui alors serait le meneur à l'assemblée ? Y a-t-il dans la Chambre Mr Papineau à part un homme tellement au-dessus des autres membres, qu'un Orateur puisse être élu sans division entre les patriotes ? (ce qui serait tant à craindre). Ceux de Québec ne manqueraient pas de vouloir élire Bédard ou Caron et ceux de Montréal Morin ou Lafontaine. Il pourrait se former quatre ou cinq partis entre les patriotes et alors tout serait perdu ... D'ailleurs parmi les patriotes un grand nombre n'est-il pas retenu par le grand nom, les grands talens, l'immense influence de $\mathrm{Mr}$ P. [apineau]. Je craindrais le départ à $P$. [apineau] plus qu'un gouverneur Dalhousien ou Aylmérien plus qu'un ministère tory. En somme nous voilà dans un bien triste état, il y a pourtant encore de grandes chances de succès. Mais suivant moi, P. [apineau] parti il n'y en aurait plus. ${ }^{22}$

La rivalité Québec-Montréal fut, à ce moment, compliquée par des dissensions à l'intérieur du clan québécois. Les mêmes difficultés se présentèrent en 1836. Debartzch, qui avait rompu

$21 \mathrm{P}$. à Huot (9 avril 1835), APQ, P-B : 524.

22 Lettre à Duvernay (4 avril 1835), APQ, coll. Duvernay. 
avec Papineau, aspirait à prendre la direction du parti canadienfrançais. Il chercha ses appuis parmi les députés de Québec. Papineau réussit toutefois à maintenir l'intégrité du parti. Mais cette quiétude fut de courte durée. L'appât des places et l'influence des femmes se combinèrent pour occasionner de nouveaux problèmes. Papineau écrivit alors à sa femme:

Tous nos avocats qui souhaitent être coiffés à tout prix, du chapeau à trois cornes, seront-ils d'une complaisance sans bornes pour nos galants commissaires? Leurs Dames ne sont point étrangères au désir de voir leurs époux porter la coiffure. Il est si doux de briller au premier rang dans les bals du Château St Louis, que l'on soupire pour la durée des beaux jours et des nuits plus belles encore, que nous procure l'arrivée de Lord Gosford. Quel plaisir de causer du dépit à celles auxquelles étaient prodiguées toutes les faveurs et les prévenances, quand nous étions négligées. Le pays est assurément mieux gouverné aux bals qu'il ne l'a jamais été ci-devant. ${ }^{23}$

A l'automne 1836, la rivalité Québec-Montréal posait de nouveaux problèmes. Il en fut de même au printemps 1837. Cette fois, il s'agissait de Lafontaine qui pensait à assumer, en s'appuyant sur le clan de Québec, la direction du parti patriote. Mais l'orientation vers la révolution déjoua ses plans. Les divergences entre Québec et Montréal persistèrent pendant tout le mouvement insurrectionnel. En 1837, les patriotes de Québec ne suivirent pas les directives du Comité central et permanent de Montréal. Mais, en 1838, ils organisèrent leur propre rébellion. Elle échoua en raison de l'intrusion d'espions bureaucrates dans l'organisation révolutionnaire.

Il serait illusoire de penser que les députés «montréalistes 》 ont manifesté une solidarité indéfectible pendant cette longue période de luttes. Nous avons la preuve que les plus dangereux riveaux de Papineau, Bourdages, Cuvillier, Mondelet, Debartzch et Lafontaine, appartenaient à la députation de la région de Montréal. Tous ont tenté d'ériger leur autorité en utilisant les sentiments locaux. Papineau avait donc des griefs personnels

23 P. à sa femme (1 février 1836), RAPQ (1953-55), 385s. 
contre Québec. Néanmoins le taux des défections demeura trop élevé dans la région de Montréal et les querelles de clans se manifestèrent à plusieurs reprises. Le clan le plus important qui comprenait les deux Nelson, Girod et Côté, apparut au début de la rébellion. Papineau lui-même n'était pas libéré de l'esprit local. Cette attitude en fit souvent un chef de clan familial ou local. Il faut signaler le clan de la Banque du Peuple auquel Papineau était particulièrement attaché. Dans ces conditions, le comportement des députés de Québec était, en partie, attribuable à Papineau qui avait tendance à s'isoler, entouré de quelques favoris, et à négliger, par crainte de se voir supplanter, des membres influents du parti. Dirigé par un chef autoritaire, le parti patriote a été secoué, d'une façon chronique, par les conflits d'intérêts personnels ou locaux. Il ne s'est maintenu que grâce à la fascination exercée par Papineau sur les masses et par la force de la conscience nationale qu'il incarnait à leurs yeux.

L'explosion de la rivalité Québec-Montréal, au moment où les classes moyennes canadiennes-françaises prétendaient vouloir organiser leur société sur le modèle démocratique, révèle combien leur expérience du parlementarisme était peu profonde. Le décalage entre la pensée et la conduite, chez les chefs patriotes, provenait surtout d'un passage trop rapide de la vie paysanne à la vie urbaine. Le goût du luxe, un besoin aigu de prestige, l'esprit de rivalité poussé à l'excès, l'influence prédominante des femmes, l'immaturité émotive et une forte dose d'empirisme étaient la caractéristique d'individus mésadaptés et, par conséquent, incapables de sacrifier pendant longtemps leurs intérêts immédiats afin de réaliser des objectifs communs. Enfin, la plupart des Canadiens français cherchaient, par le moyen des institutions démocratiques, à réaliser leur unité nationale afin de se retrouver dans l'intimité de leurs institutions traditionnelles et de leurs coutumes qui, pourtant, n'avaient rien de démocratique.

Fernand OUellet, Archives de la Province, Québec. 